\section{Optimization of ${ }^{210} \mathrm{Po}$ determination in soil}

\author{
Grzegorz Szaciłowski ${ }^{\odot}$, \\ Matgorzata Dymecka ${ }^{\circ}$, \\ Maria Prusińska (1), \\ Katarzyna Rzemek (), \\ Jakub Ośko (), \\ Magdalena Mądry (D), \\ Michalina Kostecka (1), \\ Agnieszka Araszkiewicz $\mathbb{}$
}

\begin{abstract}
In recent years, naturally occurring radioactive materials (NORM) have become an important topic from the perspective of environmental protection. The list of isotopes that should be monitored in the environment is constantly growing as new amendments to international legislation are introduced. One of the often studied NORM elements is ${ }^{210}$ Po. In this study, a process of elaborating of a new method of ${ }^{210}$ Po determination in soil was presented. In the proposed method, several analytical aspects, like the influence of silica decomposition or optimization of an electrode material, were revised. The obtained procedure allows performance of complete radiochemical analysis in a single day, with the chemical efficiency of over $85 \%$ and great reduction of costs. Further, the influence of the use of phosphate fertilizers on polonium concentration in soil was also confirmed.
\end{abstract}

Keywords: Polonium in silica $\bullet$ Polonium in soil • NORM

G. Szaciłowski ${ }^{\bowtie}$, M. Dymecka, M. Prusińska, K. Rzemek, J. Ośko, M. Mądry, M. Kostecka, A. Araszkiewicz NCBJ - National Centre for Nuclear Research Department of Nuclear Safety \& Radiation Protection A. Sołtana 7 Str., 05-400 Otwock/Świerk, Poland E-mail: grzegorz.szacilowski@ncbj.gov.pl

Received: 30 October 2020

Accepted: 7 January 2021

\section{Introduction}

Important differences in chemical properties and half-life times of elements in ${ }^{238} \mathrm{U}$ series facilitate identification of a ${ }^{210} \mathrm{~Pb}$ subseries. It consists of three elements: ${ }^{210} \mathrm{~Pb},{ }^{210} \mathrm{Bi}$, and ${ }^{210} \mathrm{Po}$, among which the greatest attention is paid to ${ }^{210} \mathrm{Po} .{ }^{210} \mathrm{Po}$ is the longest living natural isotope of polonium. It was first discovered by Maria Skłodowska-Curie and Pierre Currie in 1898. Polonium occurs widely in nature, and therefore is considered to be an important component in natural radiation background. It is estimated that about $7 \%$ of the total dose coming from ingestion of natural radionuclides comes from ${ }^{210} \mathrm{Po}$ [1]. Further, ${ }^{210} \mathrm{Po}$ (along with parent ${ }^{210} \mathrm{~Pb}$ ) can accumulate in the human food chain [2].

Polonium monitoring in the environment has over 50 years of history. Since the publication of Flynn's method of determination of ${ }^{210} \mathrm{Po}$ activity in environmental samples by spontaneous deposition on silver discs [3], the use of this element has significantly shifted to geochronology and age determination based on ${ }^{210} \mathrm{~Pb}$ (via ${ }^{210} \mathrm{Po}$ ) method. Nowadays, in the age of growing use of fertilizers in food cultivation, radiation monitoring of agricultural soil has again become an important part of radiochemistry.

The aim of this work was to elaborate a rapid, reliable, cheap, and environmentally friendly method for determination of polonium in soil as a response

0029-5922 @ 2021 The Author(s). Published by the Institute of Nuclear Chemistry and Technology.

This is an open access article under the CC BY-NC-ND 4.0 licence (http://creativecommons.org/licences/by-nc-nd/4.0/). 
to the amendment to Polish Atomic Law. The new regulations pay more attention to natural radionuclides occurring in the environment, among which polonium is considered one of the most important. To prepare the new method, several aspects of the well-known radiochemical procedure of ${ }^{210} \mathrm{Po}$ determination [4] have been revised.

\section{Experimental}

\section{Materials}

All reagents used in this work were of analytical grade and purchased from Chempur (Poland). Deionized water with conductivity less than $0.09 \mu \mathrm{S} \cdot \mathrm{cm}^{-1}$ was prepared by SolPure 78 system. Silver, copper and stainless steel discs (25 mm diameter and $0.3 \mathrm{~mm}$ thickness) were stamped from $\mathrm{Ag}_{3} \mathrm{~N}$ silver, electrolytic copper, and commercial stainless steel plates in NCBJ's workshop. The reference material IAEA-447, which contained a mixture of moss and soil from a red marble mine in Hungary, was purchased from International Atomic Energy Agency (Austria). Sample digestion was performed in MAGNUM II microwave oven from Ertec (Poland). All measurements were performed in Alpha Analyst 7200 spectrometer from Canberra (USA). The spectrometer was equipped with PIPS detectors of $1200 \mathrm{~cm}^{2}$ and $450 \mathrm{~cm}^{2}$ of active surface. Data acquisition and analysis were done by Gennie 2000 from Canberra (USA). The energy and efficiency calibration were performed by a multi-isotopic reference source from Eckert \& Zeigler (USA).

\section{Samples}

Several samples of agricultural soils were prepared to optimize the radiochemical procedure for polonium determination. The soils were sampled in early spring, when no vegetation has occurred yet. Each soil was sampled by a $10 \mathrm{~cm}$ (inner diameter) cylindrical corer, and the core was $20 \mathrm{~cm}$ high (typical depth of conventional ploughing). Samples were transferred to the laboratory in plastic bags. They were oven dried in $40^{\circ} \mathrm{C}$ in order to prevent the loss of polonium. After reaching constant weight, bulk samples were quartered into smaller, working samples (of about $10 \mathrm{~g}$ ). Further preparation consisted of grinding, sieving through $200 \mu \mathrm{m}$ mesh sieve and homogenization. Samples were stored in polypropylene vessels in ambient temperature. Further, certified material IAEA-447 was used for verification of the result.

\section{Optimization of sample decomposition}

The radiochemical procedure for polonium separation requires conversion of solid samples into solution. Among many methods (fusion, acid leaching) microwave acid digestion is considered the best for small samples. The most important advantage of microwave acid digestion is that it is a robust method which also allows time-saving and reagent consumption [5].

In order to decompose soil samples in a microwave oven, different types of digesting mixtures (typically containing $\mathrm{HNO}_{3}, \mathrm{HCl}, \mathrm{H}_{2} \mathrm{O}_{2}$, and $\mathrm{HF}$ ) are used. It is important for the mixture to contain oxidizing agents like $\mathrm{HNO}_{3}$ or $\mathrm{H}_{2} \mathrm{O}_{2}$ (to destroy organic matter and partially decompose the inorganic part of the matrix), which can be supported by inoxidizing acids like $\mathrm{HCl}$. Decomposition of silica is achieved by addition of HF; however, opinions on the influence of silica decomposition on the results remain contradictory [6].

Because of the above reason, optimization of microwave digestion has been performed. In order to determine the influence of silica decomposition on ${ }^{210} \mathrm{Po}$ results, aliquots of $0.5 \mathrm{~g}$ of five different soil samples and of one reference material were prepared. Each sample was subjected to two-step digestion, where chemical recovery of each step was controlled separately, by using two tracer isotopes $\left({ }^{208} \mathrm{Po}\right.$ and $\left.{ }^{209} \mathrm{Po}\right)$. First digestion (non-silica matrix decomposition) was performed in $8 \mathrm{~mL} \mathrm{HNO}_{3}: \mathrm{HCl}$ (3:1) mixture, the maximum temperature was set to $270^{\circ} \mathrm{C}$ and maximum pressure to $46 \mathrm{bar}$, and the process took $40 \mathrm{~min}$ to attain completion. To control chemical recovery of the first stage, isotopic tracer ${ }^{209} \mathrm{Po}$ was added into each sample prior to decomposition. The obtained solution was centrifuged and the resultant clear solution was stored for further analysis. Silicates' residue was rinsed twice with $1 \mathrm{M} \mathrm{HNO}_{3}$ and centrifuged, and each supernatant was combined with the primary solution. The residue was transferred back into a digestion vessel. To control chemical recovery of the second step of the analysis, ${ }^{208} \mathrm{Po}$ was used. The order in which tracer isotopes were used was important, as the ${ }^{208} \mathrm{Po}$ solution contained traces of ${ }^{209} \mathrm{Po}$, which is a natural impurity from the production of ${ }^{208} \mathrm{Po}$. Second digestion (silicates' decomposition) was performed in $6 \mathrm{~mL}$ of $\mathrm{HNO}_{3}: \mathrm{HF}(5: 1)$ solution under the same temperature, pressure, and time as of the first one. The product solutions of two digestions were analysed separately.

\section{Optimization of sample purification and concentration}

In case of polonium, several methods of purification and concentration are available, among which most commonly used are: fuming with $\mathrm{HCl}$ (under IR radiators), co-precipitation with $\mathrm{Fe}(\mathrm{OH})_{3}$ or $\mathrm{MnO}_{2}$, and separation on ion exchange columns [6]. The goal of this study was to achieve a rapid and cheap method, which would not result in the unnecessary production of waste.

Co-precipitation is a widely used technique which is comparably effective to fuming, but it is much less time consuming. The other advantage of this technique is that during concentration, the sample is partially purified from compounds that do not co-precipitate in these conditions. Co-precipi- 
tation can be performed with several compounds, among which $\mathrm{Ca}_{3}\left(\mathrm{PO}_{4}\right)_{2}, \mathrm{Fe}(\mathrm{OH})_{3}$, and $\mathrm{MnO}_{2}$ are the most popular.

In this study, co-precipitation with $\mathrm{MnO}_{2}$ was optimized. Although purification by $\mathrm{Fe}(\mathrm{OH})_{3}$ is more often described, it requires introducing additional $\mathrm{Fe}^{3+}$ ions, which interfere in further steps of the analysis.

Set of soil samples was mineralized and subjected to co-precipitation. Samples were transferred into $100 \mathrm{~mL}$ polypropylene centrifuge vessels and diluted by addition of $40 \mathrm{~mL}$ of deionized water. Subsequently samples were alkalized with $25 \%$ $\mathrm{NH}_{3}-\mathrm{H}_{2} \mathrm{O}$ solution until a $\mathrm{pH} \sim 9$ was reached and spiked with $4 \mathrm{~mL}$ of $0.3 \mathrm{M} \mathrm{KMnO}_{4}$. The manganese dioxide was created in situ by addition of 4-6 $\mathrm{mL}$ of $0.2 \mathrm{M} \mathrm{MnCl}_{2}$, or by addition of $1.5 \mathrm{~mL}$ of $0.6 \mathrm{M}$ $\mathrm{Na}_{2} \mathrm{SO}_{3}$. The sample was occasionally stirred for $10 \mathrm{~min}$ and centrifuged.

\section{Optimization of plating}

Generally, polonium is plated on silver, copper, or nickel discs from diluted $\mathrm{HCl}$ solutions. The nickel electrodes were eliminated from this study, as nickel has several disadvantages from the perspective of environmental protection. On the other hand, rarely described stainless steel was chosen as the promising alternative [7]. Conventional electrodes made of silver and copper were also studied.

In addition, plating on copper discs was performed in two geometries. The geometry of the vessels is described in Fig. 1.

The geometry and disc material were optimized by analysing a set of IAEA-447 samples. For geometry optimization, samples were plated on copper discs in the following way: in one set the disc was immersed into solution in a special holder (geometry A) while the sample was mounted into the vessel bottom during plating (geometry B) in the other set.

\section{Measurement}

The time of counting was diversified and depended on the sample activity. All measurements were per-

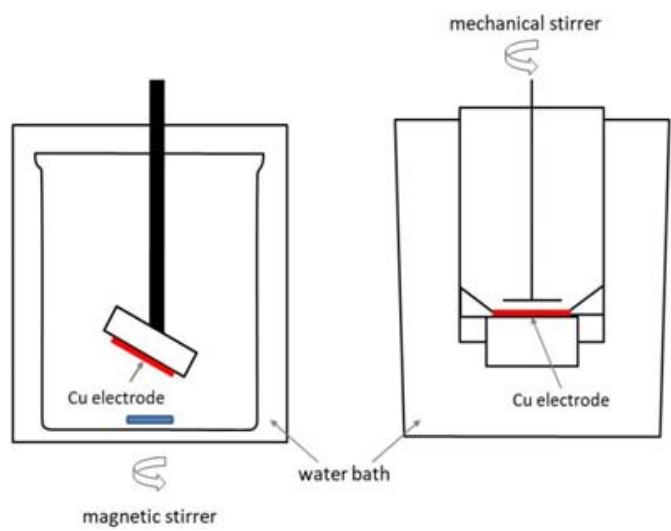

Fig. 1. Different geometries of plating, A (left) disc immersed in the solution and B (right) disc mounted to the bottom of the vessel.

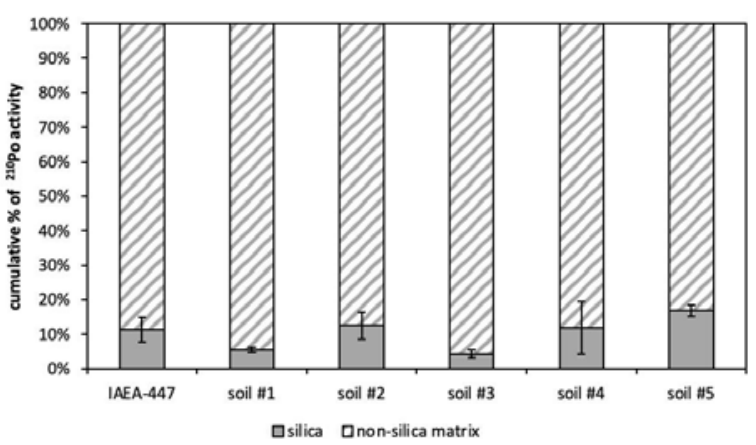

Fig. 2. Distribution of ${ }^{210} \mathrm{Po}$ between silicates and non-silica matrix in different samples.

formed with the detector of $450 \mathrm{~cm}^{2}$ of the active surface. The samples were counted until the uncertainty of peak area of analysed isotopes was below $5 \%$. Obtained activity of polonium was recalculated to the date of deposition.

\section{Results and discussion}

\section{Sample decomposition}

Two types of tracer isotopes allow us to calculate polonium concentration in silicates and non-silica matrixes separately. In order to compare analysed samples, the total ${ }^{210} \mathrm{Po}$ concentration was calculated as the sum of ${ }^{210} \mathrm{Po}$ concentration in both components of the sample. For better illustration, in Fig. 2, the results are presented as the distribution of ${ }^{210} \mathrm{Po}$ between analysed components.

The results indicate that silicates residue contains from $(4.4 \pm 1.2) \%$ to $(16.9 \pm 1.8) \%$ of polonium activity. The influence of the polonium content in silicates (defined as the percentage of the total activity) on the final result is relatively low, since it is comparable to main factors contributing to the uncertainty. However, in order to obtain results of satisfactory accuracy silicates' decomposition should be performed. Further, it was also verified through testing that in order to perform complete decomposition of the sample, one-step microwave digestion in $6 \mathrm{~mL}$ of $\mathrm{HNO}_{3}$ : $\mathrm{HF}$ solution $(5: 1)$ would be sufficient.

\section{Sample concentration and purification}

Typically, co-precipitation is achieved by creating in situ $\mathrm{MnO}_{2}$ precipitate, which is due to synproportionation between $\mathrm{MnCl}_{2}$ and $\mathrm{KMnO}_{4}$. In this study (basing on visual assessment), direct reduction of $\mathrm{KMnO}_{4}$ to $\mathrm{MnO}_{2}$ and byproducts is achieved by reaction with $\mathrm{Na}_{2} \mathrm{SO}_{3}$, and is observed to be more beneficial. In case of synproportionation, creation of $\mathrm{MnO}_{2}$ requires more time and variable amounts of $\mathrm{MnCl}_{2}$ (depending on the matrix) to obtain complete reduction of $\mathrm{KMnO}_{4}$ (indicated as the disappearance of the characteristic colour), while reduction with $\mathrm{Na}_{2} \mathrm{SO}_{3}$ proceeded instantly and allowed reduction of the amount of manganese used. It is worth men- 
tioning, that $\mathrm{Mn}^{2+}$ ions can (like iron) interfere with the plating procedure, thereby leading to degradation of the alpha spectrum.

\section{Plating}

The samples plated on stainless steel discs (Fe) revealed good full-width half-maximum (FWHM) $(20.0 \mathrm{keV})$, but the efficiency of the radiochemical procedure was very poor and below $20 \%$, which is most likely due to low plating efficiency. This significantly increased the minimum detectable activity (MDA) limit, and also resulted in very high uncertainty over the results. The results shown for $\mathrm{Ag}$ and $\mathrm{Cu}$ (plated in two geometrics) correspond well with the certified value. The source plated on Ag disc was characterized by lower FWHM (17.1 keV) and higher efficiency (over 85\%), than samples plated on $\mathrm{Cu}$ in geometry B (efficiency 60-75\%, FWHM 21.1-22.3 $\mathrm{keV})$. Results for $\mathrm{Cu}$ electrodes showed no significant differences in plating efficiency; on the other hand, the spectrum of the sample plated in geometry A is characterized by a higher FWHM value $(24.0 \mathrm{keV})$. Observation of the process and visual assessment of the obtained source allow to conclude that during plating, vapourization of the solution occurred and accumulation of bubbles on the surface of the plate could be observed. The vapour bubbles needed time to grow enough to travel upward and detach the plate, which resulted in temporary blockage of the plate surface from the solution and uneven thickness of the plated material. The surface of these sources resembled the surface of the moon. To compare the obtained results, the measured activities were recalculated to the date of deposition of last sample from this experiment. Also, the certified activity of ${ }^{210} \mathrm{Po}$ in IAEA-447 was recalculated to this date. Further, it is noteworthy to mention that, due to the long period from the certification date of IAEA-447 (over 10 years) in activity recalculation, equilibrium with ${ }^{226} \mathrm{Ra}$ and ${ }^{210} \mathrm{~Pb}$ was included.

The results of plating optimization are shown in Fig. 3.

In order to verify the results Z-score and U-score were calculated according to the IAEA publication [8]. The results are presented in Table 1.

According to Z-score tests, results for silver and both copper electrodes were satisfactory. In case of the steel electrode, the Z-score test indicated that the

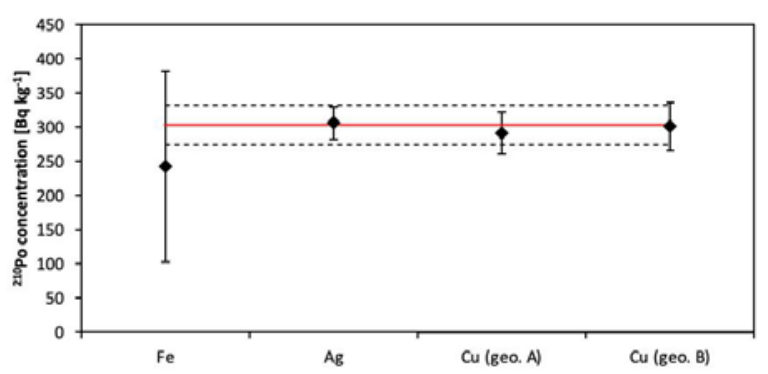

Fig. 3. Results of the ${ }^{210} \mathrm{Po}$ determination on different electrodes, the red line represents the certified value for IAEA-447 (dashed line represents the expanded uncertainty for $k=2$ of the certified value).
Table 1. Validation parameters of samples from plating optimization experiment, calculations performed for expanded uncertainty $(k=2)$

\begin{tabular}{llrr}
\hline Electrode & $\begin{array}{c}{ }^{210} \mathrm{Po} \\
\left(\mathrm{Bq} \cdot \mathrm{kg}^{-1}\right)\end{array}$ & Z-score & U-score \\
\hline $\mathrm{Fe}$ & $240 \pm 140$ & -2.11 & -0.90 \\
$\mathrm{Ag}$ & $306 \pm 24$ & 0.07 & 0.11 \\
$\mathrm{Cu}$ (geom. A) & $291 \pm 31$ & -0.61 & -0.61 \\
$\mathrm{Cu}$ (geom. B) & $301 \pm 35$ & -0.10 & -0.13 \\
\hline
\end{tabular}

results to be questionable $(2<\mid Z$-score $\mid<3)$. The $\mathrm{U}$-score test, where the uncertainty is also taken into account, produced satisfactory results in relation to all of the samples $(\mid \mathrm{U}$-score $\mid<2.58$ ).

Although the polonium plating efficiency on copper was lower than on silver, in the final procedure the $\mathrm{Cu}$ electrode and geometry B were selected, as they give satisfactory and comparable results in relation to $\mathrm{Ag}$ electrode (which is represented by obtained Z-score and U-score values). In case of $\mathrm{Cu}$ electrode, the time and acid consumption is lower, which is important from the point of view of environmental protection. Another important reason and significant advantage of the use of $\mathrm{Cu}$ is the lower price of the material, which makes the entire analysis cost-effective.

\section{Recommended radiochemical procedure}

As a result of all optimization experiments a complete procedure was elaborated (Fig. 4).

To maintain good quality alpha sources, the surface of the electrode should be free from impurities; therefore, prior to mounting, discs should be cleaned with $25 \% \mathrm{NH}_{3}-\mathrm{H}_{2} \mathrm{O}$ and immersed in $1 \mathrm{M}$ $\mathrm{HCl}$ solution for $5 \mathrm{~min}$. After deposition, the discs should be rinsed thoroughly with deionized water and stored for at least $24 \mathrm{~h}$ before counting.
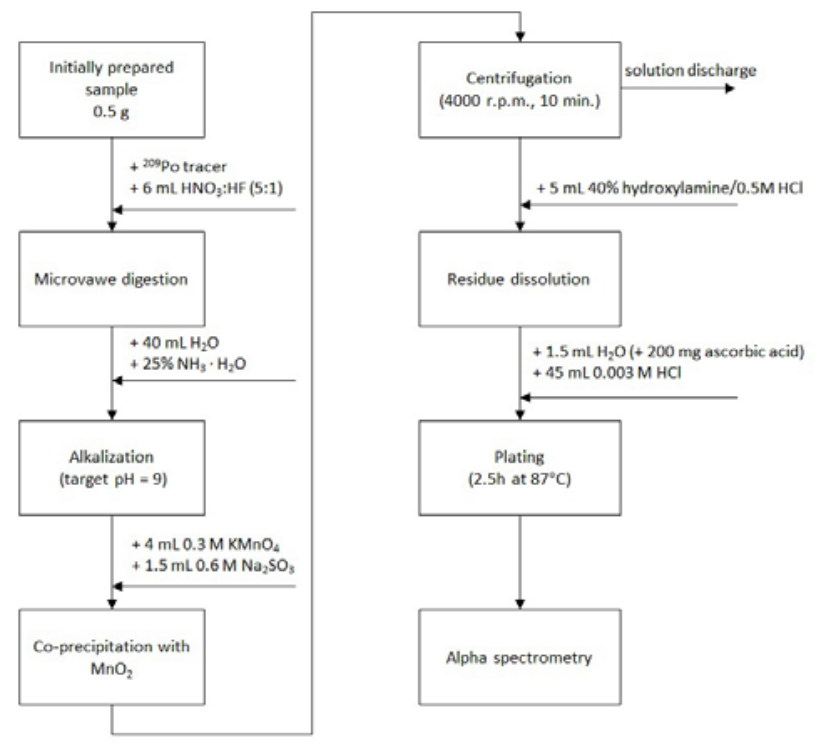

Fig. 4. Scheme of the analytical procedure for polonium determination in soil. 
Table 2. Activity concentration of ${ }^{210} \mathrm{Po}$ in soil samples

\begin{tabular}{|c|c|c|}
\hline Sample & $\begin{array}{c}{ }^{210} \mathrm{Po} \text { activity } \\
\left(\mathrm{Bq} \cdot \mathrm{kg}^{-1}\right)\end{array}$ & Type of fertilizer \\
\hline Soil 1 & $68.9 \pm 7.7$ & nitrogen \\
\hline Soil 2 & $195 \pm 12$ & phosphate \\
\hline Soil 3 & $12.1 \pm 1.8$ & nitrogen \\
\hline Soil 4 & $15.3 \pm 2.6$ & nitrogen \\
\hline
\end{tabular}

Polonium in soil determination

The radiochemical procedure which is elaborated during this study has been applied to perform determinations of ${ }^{210} \mathrm{Po}$ activity concentration in agricultural soils.

The results showed high diversity in ${ }^{210} \mathrm{Po}$ concentration in analysed soil samples (Table 2).

The soil was intentionally sampled from places intended for cultivation of different types of crops. The results indicate the presence of a clear relationship between the type of a fertilizer used and polonium concentration in soil. The samples numbers 3 and 4 represent the natural concentration of polonium in the studied area. According to literature, the mean concentration of ${ }^{226} \mathrm{Ra}$ (parent isotope to ${ }^{210} \mathrm{Po}$ ) in the studied area is about $14.3 \mathrm{~Bq} \cdot \mathrm{kg}^{-1}$ [9]. The influence of use of phosphate fertilizer on the ${ }^{210}$ Po activity can be observed for samples numbers 1 and 2 , where this type of fertilizer is currently used (sample no. 2) or was used in the past (sample no. 1).

\section{Conclusion}

In this study, a rapid and relatively cheap method for ${ }^{210} \mathrm{Po}$ in soil was elaborated. The procedure consisted of microwave digestion and co-precipitation, which allowed procurement of alpha source (from the preliminary prepared sample) in a single day. The optimization of digestion allowed determination of the influence of silicates' decomposition on the analysis of performance. To achieve accurate results, decomposition of the $\mathrm{SiO}_{2}$ should be performed.

In this method, co-precipitation with $\mathrm{MnO}_{2}$ was chosen, since it allows saving of time (in comparison to fuming technique) and is less expensive than concentration/purification on dedicated resins. The plating of polonium on either the copper or silver discs gives satisfactory results, and therefore in the proposed procedure copper discs were chosen, since choosing copper discs helped to significantly reduce the price of the analysis.

Preliminary tests of agricultural soil samples clearly indicated the relationship between type of fertilization and the radiological situation which prevails in the studied area.

\section{ORCID}

A. Araszkiewicz (D) http://orcid.org/0000-0002-2779-9006

M. Dymecka (Dhttp://orcid.org/0000-0002-0186-2185

M. Kostecka (D) http://orcid.org/0000-0001-9140-6601

M. Madry (D http://orcid.org/0000-0002-6178-8036

J. Ośko (Dttp://orcid.org/0000-0001-8966-1507

M. Prusińska (1) http://orcid.org/0000-0003-0173-9336

K. Rzemek (D) http://orcid.org/0000-0002-9387-705X

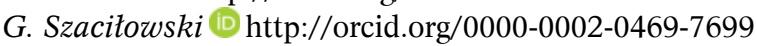

\section{References}

1. Yamamoto, M., Sakaguchi, A., Tomita, J., Imanaka, T., \& Shiraishi, K. (2009). Measurements of ${ }^{210} \mathrm{Po}$ and ${ }^{210} \mathrm{~Pb}$ in total diet samples: Estimate of dietary intakes of ${ }^{210} \mathrm{Po}$ and ${ }^{210} \mathrm{~Pb}$ for Japanese. J. Radioanal. Nucl. Chem., 279, 93-103. DOI:10.1007/s10967007-7198-8.

2. Struminska-Parulska, D., \& Olszewski, G. (2019). Is ecological food also radioecological? ${ }^{210} \mathrm{Po}$ and ${ }^{210} \mathrm{~Pb}$ studies. Chemosphere, 191, 190-195. DOI:10.1016/j. chemosphere.2017.10.051.

3. Flynn, W. W. (1968). The determination of low levels of polonium-210 in environmental materials. Anal. Chim. Acta, 43, 221-227.

4. Sethy, N. K., Sutar, A. K., Rath, P., Jha, V. N., Ravi, P. M., \& Tripathi, R. M. (2015). A review of radio chemical analysis and estimation of ${ }^{210} \mathrm{Po}$ in soil matrices. J. Radiat. Res. Appl. Sci., 8(4), 590-596. DOI:10.1016/j.jrras.2015.07.001.

5. Sun, Y. C., Chi, P. H., \& Shiue, M. Y. (2001). Comparison of different digestion methods for total decomposition of siliceous and organic environmental samples. Anal. Sci., 17(12), 1395-1399. DOI:10.2116/ analsci.17.1395.

6. Thakur, P., \& Ward, A. L. (2020) ${ }^{210}$ Po in the environment: insight into the naturally occurring polonium isotope. J. Radioanal. Nucl. Chem., 323, 27-49. DOI:10.1007/s10967-019-06939-2.

7. Johansson, L. Y. (2008). Determination of Pb-210 and Po-210 in aqueous environmental samples. Doctoral dissertation, Gottfried Wilhelm Leibniz Universität Hannover, Hannover, Germany.

8. International Atomic Energy Agency. (2012). Worldwide open proficiency. Determination of natural and artificial radionuclides in moss-soil and water. IAEA-CU-2009-03. Vienna: IAEA. (IAEA/AQ/22).

9. Isajenko, K., Piotrowska, B., Fujak, M., \& Kardaś, M. (2012). Atlas radiologiczny Polski 2011. Warszawa: Biblioteka Monitoringu Środowiska. 